\title{
Karyotype stability of human umbilical cord-derived mesenchymal stem cells during in vitro culture
}

\author{
ZHONG-BAO RUAN, LI ZHU, YI-GANG YIN and GE-CAI CHEN \\ Department of Cardiology, Taizhou People's Hospital, Taizhou, Jiangsu 225300, P.R. China
}

Received February 22, 2014; Accepted July 23, 2014

DOI: $10.3892 /$ etm.2014.1977

\begin{abstract}
The aim of this study was to investigate whether the chromosomes of human umbilical cord-derived mesenchymal stem cells (hUCMSCs) change following in vitro culture for several generations. In the present study, umbilical cords from two healthy infants following cesarean delivery were collected aseptically and hUCMSCs were isolated by digestion with collagenase and trypsin, and then cultured in vitro. hUCMSCs with fibroblastic morphology were presented from the human umbilical cord tissue after 7 days of adherent culture. When cultured for 6 passages in vitro, the hUCMSCs maintained a stable spindle-shaped morphology. Cells reached the logarithmic growth phase after 3-4 days of culture. In addition, CD13, CD29, CD44, CD90 and CD105 were highly expressed in generations P3-P6. The expression of CD31, CD34, CD45 and HLA-DR was negative. Furthermore, karyotype analysis revealed a normal diploid karyotype with 46 chromosomes and no abnormal changes were found in chromosome structure. These findings suggest that when cultured for 6 passages in vitro, hUCMSCs maintain a stable immunophenotype and chromosome structure, which provides an experimental basis for the safety of hUCMSC cytotherapy.
\end{abstract}

\section{Introduction}

Mesenchymal stem cells (MSCs) possess the capacity for self-renewal and multi-directional differentiation, and have several characteristics, including multi-lineage differentiation potential, hematopoietic support and stem cell implantation promotion, immune regulation and self-renewal, which make them a promising source for cell therapy in numerous diseases (1-3). Since MSCs are rarely found in bone marrow or fetal tissues, the isolation and expansion of human MSCs is crucial for their clinical application. Human umbilical

Correspondence to: Dr Li Zhu, Department of Cardiology, Taizhou People's Hospital, 210 Yinchun Road, Taizhou, Jiangsu 225300, P.R. China

E-mail: tzcardiac@163.com

Key words: human umbilical cord-derived mesenchymal stem cells, serial passage, karyotyping cord-derived mesenchymal stem cells (hUCMSCs) are readily available, abundant, rich in content and able to differentiate into a variety of types of cells. As a result, they have been the focus of considerable attention. However, whether the chromosomes of hUCMSCs change following culture in vitro remains unclear and controversial (4). Therefore, in the present study hUCMSCs were isolated and cultured in vitro, and the alteration in karyotypes of the hUCMSCs subjected to serial passage in vitro was determined by investigating the karyotypes of generations of P0 and P3-P6 and analysis with the G-banding technique.

\section{Materials and methods}

Materials. The materials used included DMEM/F12 medium (NVF0274; Gibco-BRL, Gaithersburg, MD, USA), fetal bovine serum (FBS; 10057; Excell Bio, Shanghai, China), collagenase II (Gibco-BRL), trypsin (Gibco-BRL), cell culture box (Nunc; Thermo Fisher Scientific, Waltham, MA, USA), flow cytometer (BD Accuri C6; BD Biosciences, Franklin Lakes, NJ, USA), inverted optical microscope (IX70-S8F; Olympus, Tokyo, Japan), mouse anti-human CD13, CD29, CD31, CD34, CD44, CD45, CD90, CD105 and HLA-DR antibodies (BD Biosciences), karyotyping system (CytoVision version 7.2; Leica, Mannheim, Germany) and a medium kit for chromosome culture (RPMI; Gibco-BRL, Grand Island, NY, USA). Colchicine, glacial acetic acid and Giemsa stain (Sigma-Aldrich, St. Louis, MO, USA) were also used.

Isolation and culture of hUCMSCs. Umbilical cords were obtained following normal or cesarean term deliveries from two healthy infants (one male and one female) at Taizhou People's Hospital (Taizhou, China) in January 2013. Written informed consent was obtained from the mothers and the experimental procedures were approved by the Ethics Committee of Taizhou People's Hospital. The umbilical cords were cannulated and washed three times with phosphate-buffered saline to remove blood clots. Then the umbilical cords were cut into 2-3 cm pieces and opened with a scalpel. The Wharton's jelly was scratched out and the vessels were removed. Thereafter, the Wharton's jelly was incubated in collagenase/DMEM solution for $24 \mathrm{~h}$. Following centrifugation for $10 \mathrm{~min}$, the cell pellet was resuspended and incubated in trypsin at $37^{\circ} \mathrm{C}$ for $30 \mathrm{~min}$. Finally, the cell pellet was resuspended in DMEM/F12 medium containing 10\% FBS and cells 


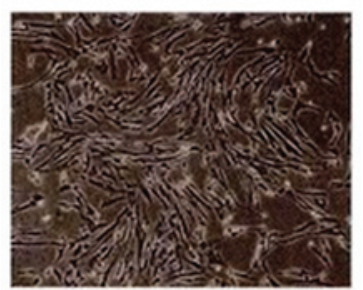

P0

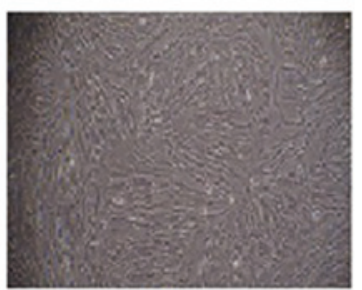

P1

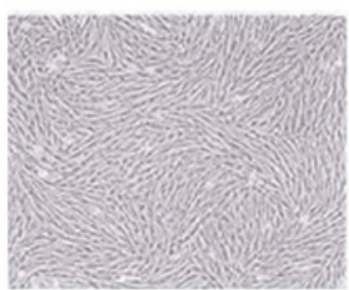

P2

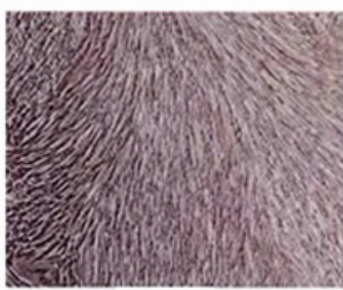

P3

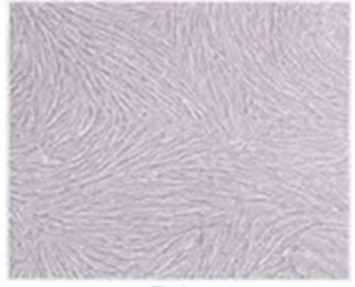

P4

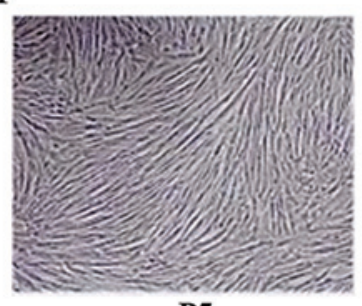

P5

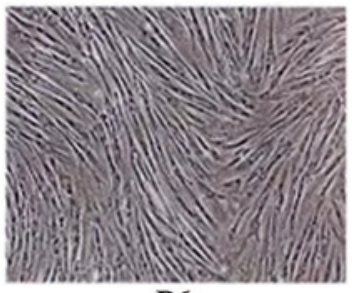

P6

Figure 1. Morphology of human umbilical cord-derived mesenchymal stem cells (inverted microscope; magnification, x40). P, passage.

were seeded in a T75 culture flask. The cells were passaged at $1 \times 10^{4}-0.4 \times 10^{6}$ cells $/ \mathrm{cm}^{2}$, and were continuously cultivated for six passages. Morphological changes were assessed by observing the cells under an inverted optical microscope.

Detection of surface markers of hUCMSCs using flow cytometry. Following the third passage (P3), the cells were washed twice with PBS, and then digested with a 1:1 mixture of trypsin $(2.5 \mathrm{~g} / \mathrm{l})$ and EDTA $(0.2 \mathrm{~g} / \mathrm{l})$. A suspension of $1 \times 10^{10}$ cells/1 was obtained by washing with PBS containing bovine serum albumin $(20 \mathrm{~g} / \mathrm{l})$. A total of $100 \mu \mathrm{l}$ cell suspension was added to each Eppendorf tube. Fluorescently labeled anti-human-antibodies [PE-CD13, PE-CD29, APC-CD90, PE-CD105, PE-CD31, PE-CD34, FITC-CD44, FITC-CD45 and FITC-HLA-DR] were added in $20 \mu \mathrm{l}$. To the control group was added immunoglobulin $\mathrm{G}$ ( $\mathrm{IgG}$; isotype control). The cells were incubated for $30 \mathrm{~min}$ at $4^{\circ} \mathrm{C}$ in the dark, washed twice with PBS, then $200 \mu \mathrm{l}$ paraformaldehyde $(10 \mathrm{~g} / \mathrm{l})$ was added to each tube. Flow cytometry was used to detect the surface markers of hUCMSCs.

Cell proliferation test. Proliferation curves were determined using the MTT method. Each passage of hUCMSCs was respectively seeded in a 96-well plate; $200 \mu \mathrm{l}$ cell suspension with a density of $2 \times 10^{4} / \mathrm{ml}$ was added to each well. The cells were incubated for $72 \mathrm{~h}$ and $10 \mu \mathrm{l}$ MTT reagent (Sigma-Aldrich) was then added to each well. The cells were incubated for a further $4 \mathrm{~h}$, the medium was removed and $200 \mu \mathrm{l}$ DMSO was added to each well to dissolve the formazan. The plates were shaken for $15 \mathrm{~min}$ at $10 \mathrm{x} \mathrm{g}$. The number of the cells per well was counted every day for 8 successive days and used to construct a proliferation curve for the hUCMSCs.

Karyotyping of generations. According to the result of the flow cytometric analysis and cell proliferation test, karyotypes were analyzed in hUCMSCs from the first passage that was isolated and cultured in vitro using the chromosome G-banding technique. The cell suspension $(300 \mu \mathrm{l})$ with a density of $2 \times 10^{4} / \mathrm{ml}$ was added to a $10-\mathrm{ml}$ culture bottle, then $100 \mu \mathrm{l}$ colchicine with a concentration of $40 \mu \mathrm{g} / \mathrm{ml}$ was added to the culture bottle. The cells were incubated for $4 \mathrm{~h}$ in a carbon dioxide incubator and the adherent cells were removed and placed into a $15-\mathrm{ml}$ centrifuge tube. Following centrifugation for $8 \mathrm{~min}$ at $182 \mathrm{x} \mathrm{g}$, the culture medium containing colchicine was removed and $4 \mathrm{ml} 0.075 \% \mathrm{KC} 1$ was added. Following incubation at $37^{\circ} \mathrm{C}$ for $5 \mathrm{~min}, 2 \mathrm{ml}$ Carnoy's solution (3:1 v/v absolute ethanol: glacial acetic acid) was added and the cells were maintained in culture at $37^{\circ} \mathrm{C}$ for $5 \mathrm{~min}$. The cells were centrifuged for a further $8 \mathrm{~min}$ at $182 \mathrm{x} \mathrm{g}$, the culture medium was removed and $4 \mathrm{ml}$ Carnoy's fixative was added. The cells were incubated and centrifuged again, which was repeated twice. The cell pellet was resuspended in DMEM/F12 medium containing 10\% FBS and aliquots of the suspension were dropped onto slides. After $48 \mathrm{~h}$ at room temperature, the slides were placed in a slide drier $75^{\circ} \mathrm{C}$ for $4 \mathrm{~h}$. Giemsa staining was then performed for $15 \mathrm{~min}$ and karyotypes of generations were analyzed using the G-banding technique.

\section{Results}

Morphology of hUCMSCs. After 7 days of adherent culture, hUCMSCs with fibroblastic morphology were presented from the human umbilical cord tissue. Following serial passage, the cells grew and proliferated quickly. When cultured for 6 passages in vitro, hUCMSCs maintained a stable spindle-shaped morphology (Fig. 1).

Immunophenotype. The results from the flow cytometric analysis revealed that standardized culture of hUCMSCs in vitro resulted in the stable expression of surface markers following serial passage. CD13, CD29, CD44, CD90 and CD105 were highly expressed at a level of $>95 \%$ on the surface of hUCMSCs, but the expression of CD31, CD34, CD45 and HLA-DR was negative and $<2 \%$ (Table I, Fig. 2).

Proliferation of hUCMSCs. Each passage of hUCMSCs presented a typical S-like proliferation curve. Cells were in a slow growth period at 1-2 days of culture, but reached a logarithmic growth phase at 3-4 days and then a plateau phase 
Table I. Immunophenotype of human umbilical cord-derived mesenchymal stem cells detected using flow cytometry (\%).

\begin{tabular}{llllllllrr}
\hline Passage & CD13 & CD29 & CD44 & CD90 & CD105 & CD31 & CD34 & CD45 & HLA-DR \\
\hline P1 & 96.70 & 98.30 & 95.60 & 99.20 & 97.10 & 0.69 & 1.24 & 0.63 \\
P2 & 97.30 & 96.40 & 96.10 & 99.30 & 95.90 & 0.18 & 0.79 & 0.51 & 0.18 \\
P3 & 99.20 & 96.90 & 95.40 & 99.80 & 95.10 & 0.71 & 1.00 & 0.24 & 0.56 \\
P4 & 95.40 & 97.20 & 97.20 & 99.60 & 96.40 & 0.62 & 0.68 & 0.72 & 0.05 \\
P5 & 98.70 & 98.60 & 96.30 & 98.70 & 96.70 & 0.34 & 0.92 & 0.29 & 0.25 \\
P6 & 96.50 & 95.10 & 95.80 & 99.10 & 98.10 & 0.59 & 0.75 & 0.46 & 0.36 \\
\hline
\end{tabular}
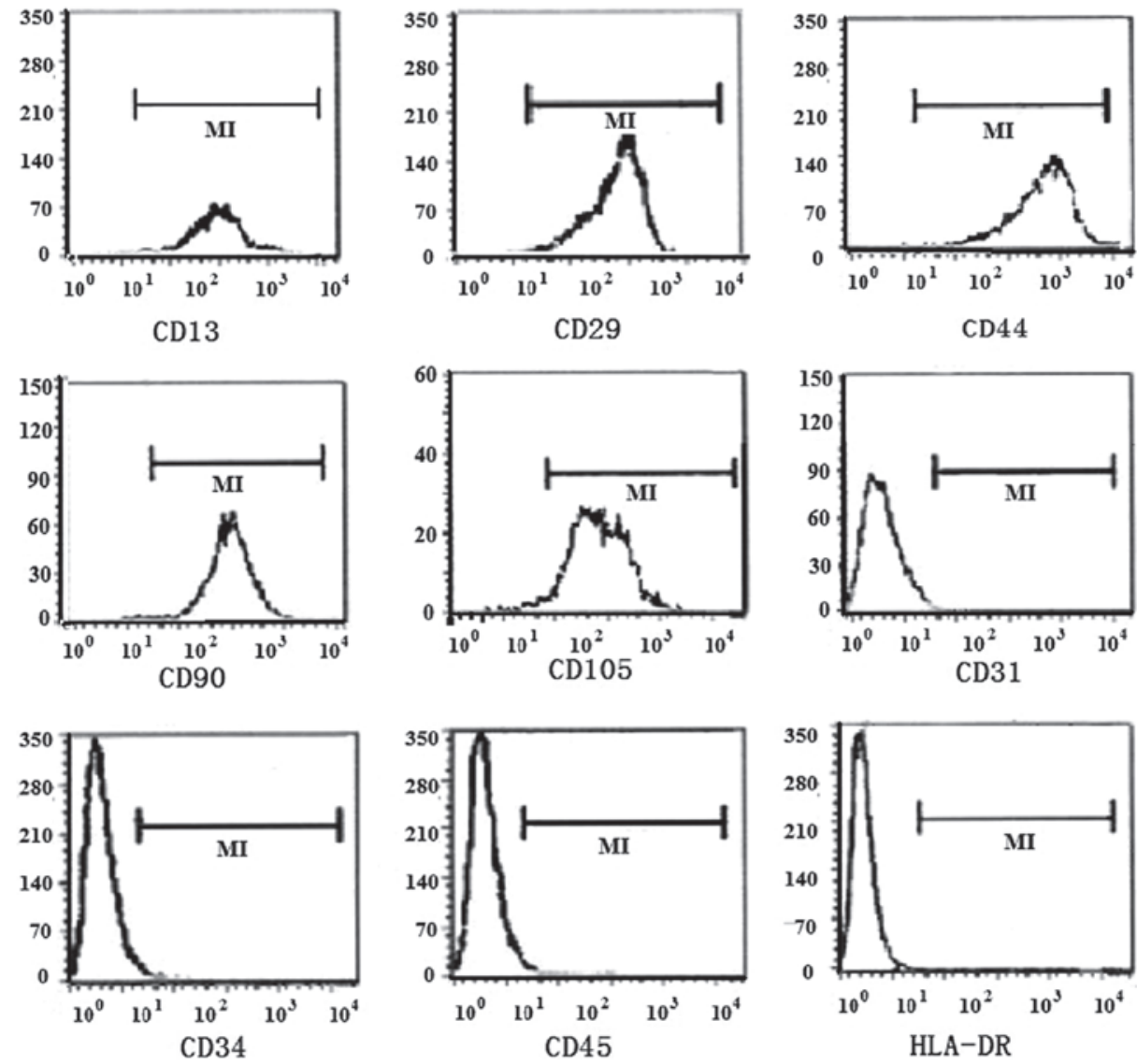

Figure 2. Immunophenotype results of human umbilical cord-derived mesenchymal stem cells.

at 5-7 days. After 8 days of culture, the cells were observed to have diminished proliferation potency (Fig. 3).

Karyotype analysis of hUCMSCs. Following treatment with colchicine, P3-P6 of hUCMSCs were arrested in the metaphase stage. A normal diploid karyotype with 46 chromosomes and no abnormal changes in chromosome structure was observed by the analysis of 30 metaphase cells (Fig. 4). However, abnormal morphology was observed in the seventh and eighth passages, and the ratio of non-hUCMSCs also increased in these passages.

\section{Discussion}

MSCs offer a lot of promise for the development of novel alternative cell-based therapies. These unique cells possess two major features: their ability for self-renewal and differentiation potential. At present, MSCs are primarily obtained from bone marrow. However, bone marrow-derived MSCs are easily contaminated by viruses and have a significantly decreased capacity for proliferation and differentiation with the aging of the donor. In addition, as there are a variety of ethical and legal issues, their application is restrained $(5,6)$. Umbilical cord blood (UCB) is a source of additional stem cells for experimental and potentially clinical uses. However, the presence of MSCs in UCB is controversial (7). Human umbilical cord, a connecting tissue of extraembryonic origin lying between the mother and fetus, consists of two arteries, one vein, inter-vessel connective tissue and umbilical epithelium. The connective tissue, also referred as Wharton's jelly, is composed of a sponge-like structure woven by collagen fibers, 

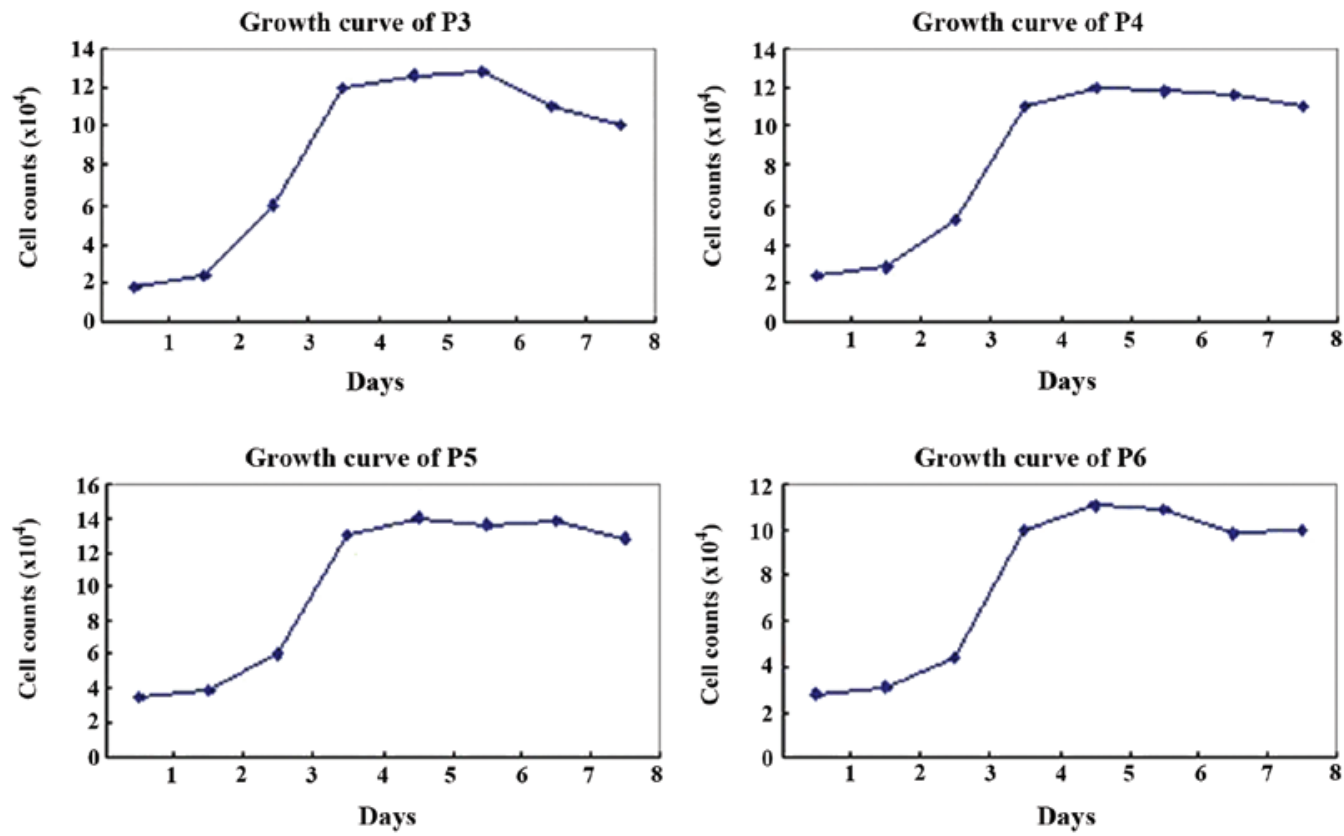

Figure 3. Growth curves of human umbilical cord derived mesenchymal stem cells. P, passage.

\section{Male}

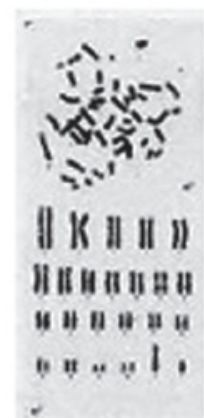

P0

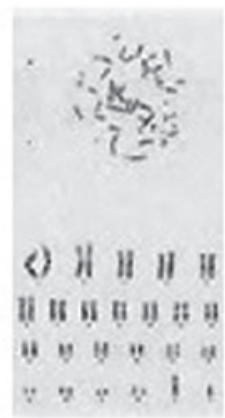

P3

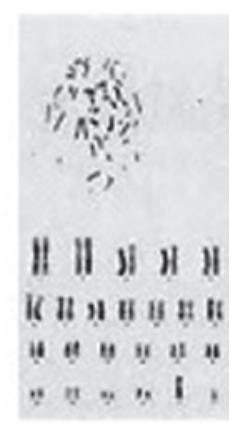

P4

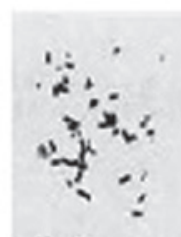

|| || || || |

(

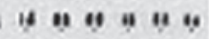

$\because * n+1$.

P5

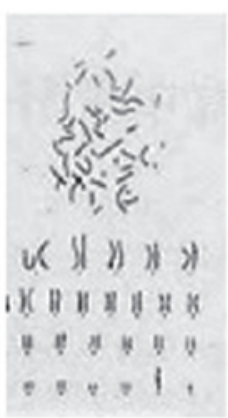

P6

\section{Female}

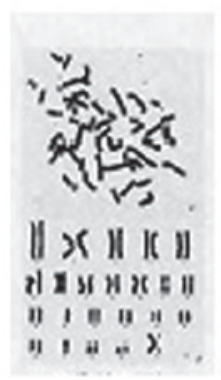

P0

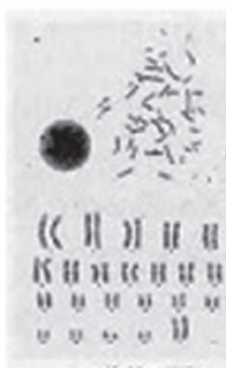

P3

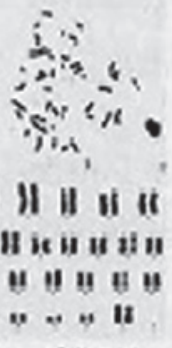

P4
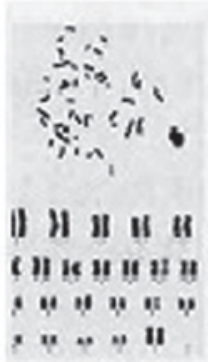

P5

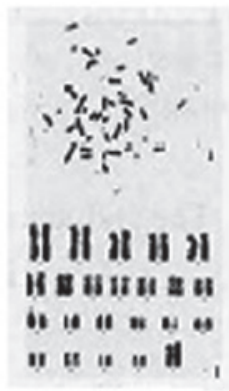

P6

Figure 4. Karyotype analisis of human umbilical cord derived mesenchymal stem cells. P, passage.

proteoglycan and embedded stromal cells. The umbilical cord is a promising source of MSCs due to its wide availability, differentiation potential and lack of ethical concerns. Compared with bone marrow MSCs, hUCMSCs are easier to isolate and expand, and so they would be an advantageous, novel source of adult MSCs. A number of studies have demonstrated that hUCMSCs may have an important role in the application and experimental research of adult human MSCs $(8,9)$.

Due to differences between culture systems in vitro and conditions in vivo, whether MSCs maintain a stable chromo- 
some structure following serial passage and proliferation is an important index of clinical safety. Numerous studies have found that an abnormal chromosome structure is positively correlated with the incidence of tumors $(10,11)$. The results of investigations of the karyotype stability of MSCs during a long-term culture in vitro remain inconsistent. It has been demonstrated that MSCs passaged >10 times have a chromosome analysis that is normal $(4,12)$; however, other studies have found that karyotype variation in MSCs may occur following long-term culture in vitro and have tumorigenicity in nude mice (13-15). Although hUMSCs are a desirable source of cells for use in tissue repair and regeneration engineering, few studies have addressed the safety of hUMSCs that have been subjected to long-term culture in vitro.

In the present study, by digestion with collagenase and trypsin, a large number of adherent hUMSCs were harvested within a short time period. The results showed that following resuscitation, these cells maintain high cell viability, and could be cultured for a long time, enabling large numbers of cells to be harvested. The results from the flow cytometric analysis demonstrated that the hUCMSCs harvested in the present study maintained their phenotypes following long-term in vitro proliferation and serial passage. CD13, CD29, CD44, CD90 and CD105 were highly expressed on $>95 \%$ of the surface of hUCMSCs; however, the expression of CD31,CD34, CD45 and HLA-DR was negative and $<2 \%$, which is consistent with a previous study (16). The results from the proliferation test demonstrated that each passage of hUCMSCs presented a typical S-like proliferation curve. Cells were in a logarithmic growth phase after 3-4 days of culture. According to the results of proliferation test, the hUCMSCs of P3-P6 were selected, which were then cultured for 3-4 days for karyotyping. The karyotype analysis showed a normal diploid karyotype with 46 chromosomes and no abnormal changes were observed in chromosome structure in the hUCMSCs of P3-P6 following treatment with colchicine. In the present study, it was found that there was abnormal morphology in the seventh and eighth passages and the ratio of non-hUCMSCs was also increased in these passages.

In conclusion, the present study demonstrated that hUCMSCs maintain a stable immunophenotype and chromosome structure when cultured for 6 passages in vitro, which provides an experimental basis for the safety of hUCMSC cytotherapy.

There were a number of limitations in the present study. The number of the subjects in this study was small, which may have affected the reliability of the results. The karyotype stability of hUCMSCs was not evaluated following long-term culture. It remains to be determined whether the chromosomes of hUCMSCs change following in vitro culture for larger numbers of generations and over a longer period of time in future studies.

\section{References}

1. Tong CK, Vellasamy S, Tan BC, Abdullah M, Vidyadaran S, Seow HF and Ramasamy R: Generation of mesenchymal stem cell from human umbilical cord tissue using a combination and mechanical disassociation method. Cell Biol Int 35: 221-226, 2011.

2. Arufe MC, De la Fuente A, Fuentes I, Toro FJ and Blanco FJ: Umbilical cord as a mesenchymal stem cell source for treating joint pathologies. World J Orthop 2: 43-50, 2011.

3. Méndez-Ferrer S, Michurina TV, Ferraro F, Mazloom AR, Macarthur BD, Lira SA, Scadden DT, Ma'ayan A, Enikolopov GN and Frenette PS: Mesenchymal and haematopoietic stem cells form a unique bone marrow niche. Nature 466: 829-834, 2010.

4. Montanucci P, Basta G, Pescara T, Pennoni I, Di Giovanni F and Calafiore R: New simple and rapid method for purification of mesenchymal stem cells from the human umbilical cord Wharton jelly. Tissue Eng Part A 17: 2651-2661, 2011.

5. Stewart MC and Stewart AA: Mesenchymal stem cells: characteristics, sources, and mechanisms of action.Vet Clin N Am Equine Pract 27: 243-261, 2011.

6. Ringden O and Le Blanc K: Mesenchymal stem cells for treatment of acute and chronic graft-versus-host disease, tissue toxicity and hemorrhages. Best Pract Res Clin Haematol 24: 65-72, 2011.

7. Kern S, Eichler H, Stoeve J, Klüter H and Bieback K: Comparative analysis of mesenchymal stem cells from bone marrow, umbilical cord blood, or adipose tissue. Stem Cells 24: 1294-1301, 2006.

8. Cho H, Seo YK, Jeon S, Yoon HH, Choi YK and Park JK: Neural differentiation of umbilical cord mesenchymal stem cells by sub-sonic vibration. Life Sci 90: 591-599, 2012.

9. Matsuzuka T, Rachakatla RS, Doi C, Maurya DK, Ohta N, Kawabata A, Pyle MM, Pickel L, Reischman J, Marini F, Troyer D and Tamura M: Human umbilical cord matrix-derived stem cells expressing interferon-beta gene significantly attenuate bronchioloalveolar carcinoma xenografts in SCID mice. Lung Cancer 70: 28-36, 2010.

10. Tolar J, Nauta AJ, Osborn MJ, Panoskaltsis Mortari A, McElmurry RT, Bell S, Xia L, Zhou N, Riddle M, Schroeder TM, Westendorf JJ, McIvor RS, Hogendoorn PC, Szuhai K, Oseth L, Hirsch B, Yant SR, Kay MA, Peister A, Prockop DJ, Fibbe WE and Blazar BR:Sarcoma derived from cultured mesenchymal stem cells. Stem Cells 25: 371-379, 2007.

11. Bagley RG, Weber W, Rouleau C, Yao M, Honma N, Kataoka S, Ishida I, Roberts BL and Teicher BA: Human mesenchymal stem cells from bone marrow express tumor endothelial and stromal markers. Int J Oncol 34: 619-627, 2009.

12. Fotino C, Ricordi C, Lauriola V, Alejandro R and Pileggi A: Bone marrow-derived stem cell transplantation for the treatment of insulin dependent diabetes. Rev Diabet Stud 7: 144-157, 2010.

13. Tolar J, Nauta AJ, Osborn MJ, Panoskaltsis Mortari A, McElmurry RT, Bell S, Xia L, Zhou N, Riddle M, Schroeder TM, Westendorf JJ, McIvor RS, Hogendoorn PC, Szuhai K, Oseth L, Hirsch B, Yant SR, Kay MA, Peister A, Prockop DJ, Fibbe WE and Blazar BR: Sarcoma derived from cultured mesenchymal stem cells.Stem Cells 25: 371-379, 2007.

14. Tasso R, Augello A, Carida' M, Postiglione F, Tibiletti MG, Bernasconi B, Astigiano S, Fais F, Truini M, Cancedda R and Pennesi G: Development of sarcomas in mice implanted with mesenchymal stemcells seeded onto bioscaffolds. Carcinogenesis 30: 150-157, 2009.

15. Li Q, Hisha H, Takaki T, Adachi Y, Li M, Song CH, Feng W, Okazaki S, Mizokami T, Kato J, Inaba M, Hosaka N, Maki M and Ikehara S: Trabsformation potential of bone marrow stromal cells into undifferentiated high-grade pleomorphic sarcoma. J Canc Res Clin Oncol 136: 829-838, 2010.

16. Chabannes D, Hill M, Merieau E, Rossignol J, Brion R, Soulillou JP, Anegon I and Cuturi CM: A role for heme oxygenase-1 in the immunosuppressive effect of adult rat and human mesenchymal stem cells. Blood 110: 3691-3694, 2007 